\title{
Dynamic Analysis of Stockbridge Damper
}

\author{
Nilson Barbieri ${ }^{1,2}$ and Renato Barbieri ${ }^{1}$ \\ ${ }^{1}$ Mechanial Engineering Department, Pontifícia Universidade Católica do Paraná (PUCPR), Rua Imaculada Conceição, \\ 1155, 80215-901 Curitiba, PR, Brazil \\ ${ }^{2}$ Mechanial Engineering Department, Universidade Tecnológica Federal do Paraná (UTFPR), Rua Sete de Setembro, \\ 3165, 80230-901 Curitiba, PR, Brazil
}

Correspondence should be addressed to Nilson Barbieri, nilson.barbieri@pucpr.br

Received 9 April 2012; Accepted 18 May 2012

Academic Editor: Joseph C. S. Lai

Copyright (c) 2012 N. Barbieri and R. Barbieri. This is an open access article distributed under the Creative Commons Attribution License, which permits unrestricted use, distribution, and reproduction in any medium, provided the original work is properly cited.

\begin{abstract}
In this work a machine cam with five different profiles was used to investigate the linear and nonlinear dynamical behavior of asymmetric Stockbridge damper with excitation frequencies in the range of $5 \leq f \leq 17 \mathrm{~Hz}$. The experimental vibration signals were acquired through accelerometers placed along the sample. The loss factor and the Young's modulus were estimated through approximation of the experimental and numerical results using Genetic Algorithms (GAs). Linear and nonlinear mathematical models were used to adjust the data. The two parameters are dependent on the excitation frequency and the amplitude of the base displacement. The results are validated comparing typical impedance curves obtained in conventional testing using an electromechanical shaker.
\end{abstract}

\section{Introduction}

The Stockbridge damper is presently the most common type of transmission line damper. In general, the absorber consists of two weights attached to the end of stranded cables, which are known as messenger wires.

A detailed mathematical description of conductor motion is difficult due to the stranded construction of a conductor. An example of this problem is the study carried out by Nawrocki and Labrosse [1], where the cable is modeled using each individual wire model and all possible contacts are investigated. However, in order to get good results for the static analysis, this model was not applied for dynamic problems and the dynamic friction between the individual wires of the cable was also not studied.

In Stockbridge dampers of transmission line, mechanical energy is dissipated in wire cables "damper or messenger cables". The damping mechanism is due to statical hysteresis resulting from Coulomb (dry) friction between the individual wires of the cable undergoing bending deformation. In order to test this dynamical model of Stockbridge damper, the typical experimental impedance curves are compared with numeric results. This procedure was used by [2] who uses the masing model for modeling the nonlinear damping behavior of the damper cable of the Stockbridge damper.

Zhu and Meguid [3-5] analyzed the behavior of cables considering curved beam models. Due to difficulties in modeling the hysteretic damping, the standard Rayleigh damping was used. The numerical and experimental results presented good approximation.

López and Venegas [6] analyzed the dynamic behavior of Stockbridge dampers through dynamic and fatigue tests and showed that the damping ratio decreases linearly with the excitation motion amplitude.

In this work, the physical parameters are adjusted through the comparison between numerical and experimental results. The numerical models are obtained using the Finite Element Method. The experimental results are obtained using a cam machine with five different eccentricities. This way, the motion amplitude of the excitation is maintained constant. The experimental results are compared with linear and nonlinear numerical results. The nonlinear system contains nonlinear stiffness and damping elements. The dynamic responses of the linear and nonlinear systems are 


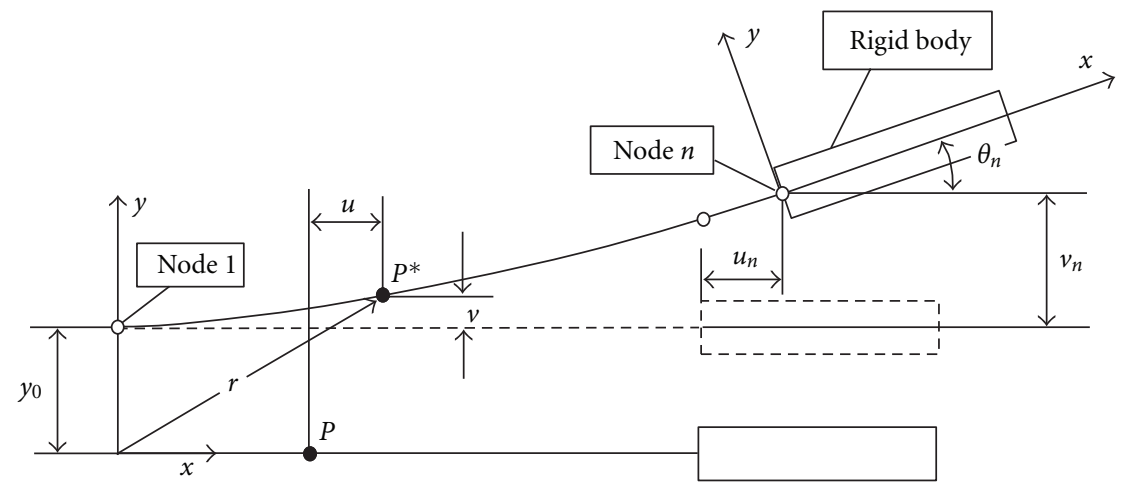

Figure 1: References and admissible displacements.

also compared with the experimental results (impedance curves) obtained through conventional testing using an electromechanical shaker to excite the system.

\section{Mathematical Models}

The mathematical models of the Stockbridge damper system are shown in this section.

2.1. Messenger Wire Model. The messenger wire is modeled by the Euler-Bernoulli beam finite element. In this element, the transversal displacement is interpolated using the wellknown Hermitian interpolation polynomials with $C^{1}$ continuity, and the degrees of freedom (d.o.f) in each node are the transversal displacement and the rotation, $\{v, \theta\}$. The dynamic equation for this element can be written as:

$$
\begin{aligned}
& \frac{\rho A L}{420}\left[\begin{array}{cccc}
156 & 22 L & 54 & -13 L \\
& 4 L^{2} & 13 L & -3 L^{2} \\
\text { sym. } & 156 & -22 L \\
& & 4 L^{2}
\end{array}\right]\left\{\begin{array}{l}
\ddot{v}_{1} \\
\ddot{\theta}_{1} \\
\ddot{v}_{2} \\
\ddot{\theta}_{2}
\end{array}\right\} \\
& +\frac{E I}{L^{3}}\left[\begin{array}{cccc}
12 & 6 L & -12 & 6 L \\
& 4 L^{2} & -6 L & 2 L^{2} \\
\text { sym. } & & 12 & -6 L \\
& & 4 L^{2}
\end{array}\right]\left\{\begin{array}{l}
v_{1} \\
\theta_{1} \\
v_{2} \\
\theta_{2}
\end{array}\right\}=-\frac{\rho A L}{12}\left\{\begin{array}{c}
6 \\
L \\
6 \\
-L
\end{array}\right\} \ddot{y}_{o},
\end{aligned}
$$

where $\rho A$ is the linear density of the cable, $L$ is the finite element length, $E I$ is the cable flexural (bending) stiffness and $\ddot{y}_{o}$ is the base acceleration (the acceleration imposed by the cam machine).

In order to take into consideration the cable hysteretic damping in (1), it is enough to consider the flexural stiffness as [6];

$$
E I=E I_{o}(1+\eta i)
$$

where $\eta$ is the hysteretic damping constant and $i=\sqrt{-1}$.

2.2. Damper Mass Model. The suspended masses of the Stockbridge damper are modeled with a rigid body plane motion hypothesis and the admissible displacements are shown in Figure 1.
After assembling all the elements of the messenger wire, each weight of the Stockbridge damper contributes to two terms of the dynamical equilibrium. The first contribution is in the mass matrix (inertia force)

$$
\left[M_{S}\right]\left\{\ddot{q}_{n}\right\}=\left[\begin{array}{cc}
m & m \bar{x} \\
m \bar{x} & I_{n}
\end{array}\right]\left\{\begin{array}{c}
\ddot{v}_{n} \\
\ddot{\theta}_{n}
\end{array}\right\}
$$

and another parcel is in the vector force due to base acceleration

$$
\left\{f_{s}\right\}=-\left\{\begin{array}{c}
m \\
m \bar{x}
\end{array}\right\} \ddot{y}_{o},
$$

where $m$ is the mass of the Stockbridge damper weight, $\bar{x}$ is the center of mass coordinate and $I_{n}$ is the inertia moment with the reference fixed in node $n$.

These two terms are obtained using the first variation of the kinetic energy (Hamilton Principles) and the rigid body plane motion hypothesis for modeling the suspended Stockbridge damper weight. With the convention defined in Figure 1, the kinetic energy of each weight of the Stockbridge damper can be written by taking node $n$ (the node of the finite element mesh connected to the messenger wire) as a reference. This expression is

$$
T=\frac{1}{2} m \dot{r}_{n} \cdot \dot{r}_{n}+\dot{r}_{n} \cdot[\omega \times m \bar{x}]+\frac{1}{2} \omega \cdot \int_{m} \rho_{n} \times\left[\omega \times \rho_{n}\right] d m,
$$

where $\dot{r}_{n}$ is the velocity of node $n, \omega$ is the damper weight angular velocity and $\rho \mathrm{n}$ is the particle position of $d m$ mass with the origin fixed in node $n$.

Taking into consideration the hypotheses of plane motion of rigid body, $\omega=\dot{\theta}_{n} k$, and symmetry in $y,(\bar{y}=0)$, the expression for the damper weight kinetic energy can be rewritten after integration as

$$
\begin{aligned}
T= & \frac{1}{2} m\left[\dot{u}_{n}^{2}+\dot{v}_{n}^{2}+2 \dot{y}_{0} \dot{v}_{n}+\dot{y}_{0}^{2}\right] \\
& +\bar{x} m\left[\dot{\theta}_{n} \dot{v}_{n}+\dot{\theta}_{n} \dot{y}_{0}\right]+\frac{1}{2} I_{n} \dot{\theta}_{n}^{2} .
\end{aligned}
$$

The variation of $T$ can be written as $\delta T=\partial T / \partial \dot{u}_{n} \delta \dot{u}_{n}+$ $\partial T / \partial \dot{v}_{n} \delta \dot{v}_{n}+\partial T / \partial \dot{\theta}_{n} \delta \dot{\theta}_{n}$. Integrating by parts the terms in $\delta \dot{v}_{n}$ and $\delta \dot{\theta}_{n}$ and neglecting the boundary terms, it is found in (3) and (4). 
2.3. Finite-Element System of Equations. The Stockbridge damper dynamical equilibrium equation is obtained after assembling all finite elements and it can be conventionally written as

$$
[M]\{\ddot{q}\}+[K]\{q\}=\left\{f_{o}\right\} \ddot{y}_{o}(t)
$$

where $[M]$ and $[K]$ are the mass and stiffness matrices, respectively, and $\left\{f_{o}\right\}$ is the force vector. The vector components $\{q\}$ are the finite-element node displacements and rotations, $v$ and $\theta$ and $\ddot{y}_{o}$ is the acceleration in node 1 (base shaker acceleration).

Considering the base excitation as harmonic, $\ddot{y}_{o}(t)=$ $\left|\ddot{y}_{o}\right| e^{i \omega t}$, the solution $q(t)$ becomes $q(t)=q_{o} e^{i \omega t}$. Substituting this supposed solution into the motion equation (7) yields

$$
\left[-\omega^{2}[M]+[K]\right]\left\{q_{o}\right\}=\left|\ddot{y}_{o}\right|\left\{f_{o}\right\} .
$$

The amplitude of the displacement vector is calculated solving Equation (8) for each frequency $\omega$ and the amplitude of the acceleration vector is easily calculated with the product of $\omega^{2}\left\{q_{o}\right\}$.

2.4. The Nonlinear Formulation. The parameter estimation of the nonlinear system can be made by approximating the numeric and experimental Frequency Response Function (FRF) curves. The analysis can be exemplified considering the motion equation of a simple oscillator subjected to a harmonic excitation [7-11] (Harmonic Balance Method):

$$
m \ddot{y}+\tilde{g}(\dot{y}, y)=f \sin \omega t
$$

where $\tilde{g}(\dot{y}, y)$ encloses all the restoring forces and supposing it is a nonlinear function of the velocity and displacement $(\dot{y}, y)$ of the mass $m$.

It is possible to find a linearized coefficient $\tilde{v}(\dot{y}, y)$ which provides the best average of the true restoring force. This coefficient acts on the fundamental harmonic of the nonlinear response $\left(\tilde{Y}^{1 s t}\right)$ for a single load cycle, in such way that [8]

$$
\tilde{g}(\dot{y}, y) \approx \tilde{\nu}(\dot{y}, y) \cdot y, \quad \text { for } y \approx \tilde{Y}^{1 s t} \sin (\omega t+\theta)=\tilde{Y}^{1 s t} \sin \tau .
$$

In order to find the nonlinear coefficient $\tilde{\nu}(\dot{y}, y)$, the restoring force $\tilde{g}(\dot{y}, y)$ is expanded around $y$ via a Fourier series, neglecting all the higher order terms:

$$
\begin{aligned}
\tilde{g}(\dot{y}, y) \approx \tilde{v}(\dot{y}, y) \cdot y= & \sigma_{a}^{1 \mathrm{st}} y+\sigma_{b}^{1 \mathrm{st}} y \\
& +\sigma_{c}^{1 \mathrm{st}} y+\sigma_{d}^{1 \mathrm{st}} y+\cdots,
\end{aligned}
$$

where the $\sigma$ functions are given by

$$
\begin{aligned}
& \sigma_{a}^{1 \mathrm{st}}=\frac{1}{\pi \tilde{Y}^{1 \mathrm{st}}} \int_{0}^{2 \pi} \tilde{g}\left(\tilde{Y}^{1 \mathrm{st}} \sin \tau, \omega \tilde{Y}^{1 \mathrm{st}} \cos \tau\right) \sin \tau d \tau, \\
& \sigma_{a}^{1 \mathrm{st}}=\frac{1}{\pi \tilde{Y}^{1 \mathrm{st}}} \int_{0}^{2 \pi} \tilde{g}\left(\tilde{Y}^{1 \mathrm{st}} \sin \tau, \omega \tilde{Y}^{1 \mathrm{st}} \cos \tau\right) \cos \tau d \tau .
\end{aligned}
$$

The nonlinear coefficient $\tilde{v}(\dot{y}, y)$ is defined by (using only the first two terms of (11)):

$$
\tilde{v}(\dot{y}, y)=\sigma_{a}^{1 s t}+\sigma_{b}^{1 s t} .
$$

The mathematical model of a cubic stiffness element can be expressed as [8]:

$$
\tilde{g}_{k}(\dot{y}, y)=k y+\beta y^{3},
$$

where the coefficient $k$ represents the linear component of the spring and the coefficient $\beta$ accounts for the nonlinear effects due to the term $y^{3}$. Introducing (15) into (12) and (13) and dropping the superscript first and after many manipulations, it can be found that the first-order representation of a cubic stiffness element is [8]:

$$
\tilde{\nu}_{k}(\dot{y}, y)=k+\frac{3 \beta \tilde{Y}^{2}}{4},
$$

where the second term on the right side of (16) represents the nonlinear part of the coefficient.

The nonlinear friction damping can be obtained using a similar approach to the cubic stiffness development. The nonlinear restoring force becomes

$$
\tilde{g}_{d}(\dot{y}, y)=c \dot{y}+\gamma \frac{\dot{y}}{|\dot{y}|} .
$$

The linearized coefficient $\tilde{v}_{c}(\dot{y}, y)$ is found to be

$$
\tilde{v}_{c}(\dot{y}, y)=i \omega c+i \frac{4 \gamma}{\pi \tilde{Y}} .
$$

The $\tilde{g}(\dot{y}, y)$ restoring forces presents in (9) involves the sum of (15) and (17).

Expanding the idea of the simple oscillator introduced in (9) to a MDOF system, we have

$$
[M]\{\ddot{y}\}+\{\widetilde{G}(\dot{y}, y)\}=\{F\} e^{i \omega t},
$$

where $[M]$ is the mass matrix; $\{\ddot{y}\},\{\dot{y}\}$ and $\{y\}$ are the acceleration, velocity and displacement vectors, respectively, and $\{F\}$ is the harmonic excitation vector operating at frequency $\omega$.

For additive nonlinearities, it is possible to expand the nonlinear vector into individual nonlinear restoring forces, as follows:

$$
\{\tilde{G}(\dot{y}, y)\}=\left\{\begin{array}{c}
\tilde{g}_{11}+\widetilde{g}_{12}+\widetilde{g}_{13}+\cdots+\widetilde{g}_{1 N} \\
\widetilde{g}_{21}+\widetilde{g}_{22}+\widetilde{g}_{23}+\cdots+\widetilde{g}_{2 N} \\
\widetilde{g}_{31}+\widetilde{g}_{32}+\widetilde{g}_{33}+\cdots+\widetilde{g}_{3 N} \\
\cdots \\
\tilde{g}_{N 1}+\tilde{g}_{N 2}+\tilde{g}_{N 3}+\cdots+\widetilde{g}_{N N}
\end{array}\right\},
$$

where $N$ is the size of the system (in DOFs). Each nonlinear function $\tilde{g}_{i j}$ represents a restoring force acting between DOFs $i$ and $j$, while terms with repeated indexes $\tilde{g}_{i i}$ represent a restoring force between DOF $i$ and the ground.

Introducing the newly redefined nonlinear coefficients into (20), a matrix of nonlinear coefficients is formed:

$$
\{\tilde{G}(\dot{y}, y)\}=[\tilde{v}]\{\tilde{Y}\} .
$$




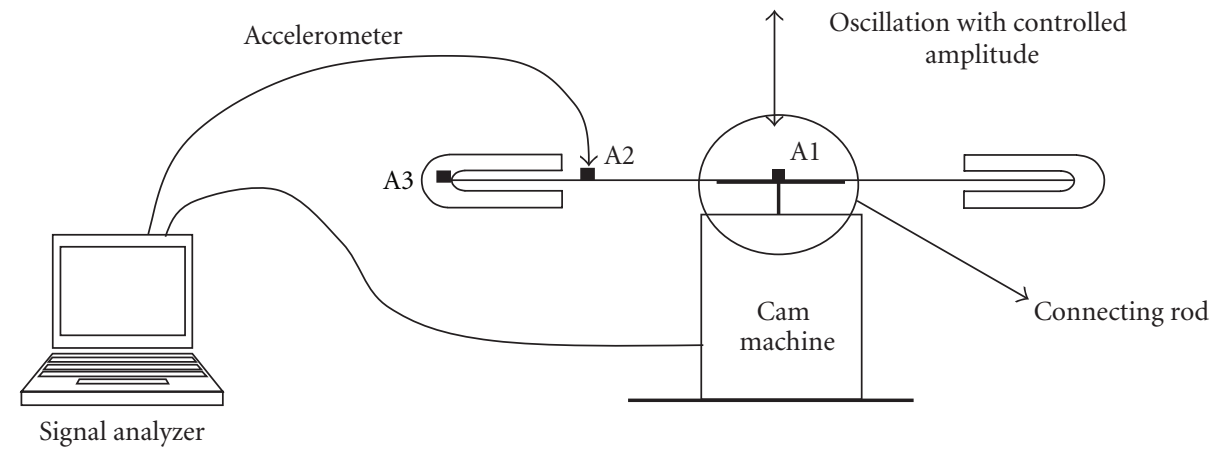

FIgURE 2: Schematic cam machine.

The motion equation of a general nonlinear system subjected to harmonic excitation can be described by the following nonlinear ordinary differential equation:

$$
\begin{aligned}
& {[M]\{\ddot{y}\}+[C]\{\dot{y}\}+i[D]\{y\} } \\
&+[K]\{y\}+\{\tilde{G}(\dot{y}, y)\}=\{F\} e^{i \omega t},
\end{aligned}
$$

where $[M],[C],[D]$, and $[K]$ are the mass, viscous damping, hysteretic damping, and stiffness matrices, respectively, of the underlying linear system; $\{\ddot{y}\},\{\dot{y}\}$, and $\{y\}$ are the acceleration, velocity and displacement vectors, respectively, and $\{F\}$ is the harmonic excitation vector operating at frequency $\omega$.

The nonlinear component of the system is represented by the nonlinear vector $\{\widetilde{G}\}$, which is a function of all displacements and velocities in the general case.

Considering a harmonic response $\{y(t)\}=\{\tilde{Y}\} e^{\mathrm{i} \omega \mathrm{t}}$, where $\{\tilde{Y}\}=\left\{|\tilde{Y}| e^{i \theta}\right\}$ is a nonlinear complex vector allowing it to accommodate phase, the motion equation is further reduced to

$$
\left(-\omega^{2}[M]+i \omega[C]+i[D]+[K]\right)\{\tilde{Y}\}+\{\tilde{G}(\omega, \tilde{Y})\}=\{F\} .
$$

The linear receptance can be defined as

$$
[\alpha]=\left(-\omega^{2}[M]+i \omega[C]+i[D]+[K]\right)^{-1}
$$

and its inverse, $[\Lambda]=[\alpha]^{-1}$, as

$$
[\Lambda]=-\omega^{2}[M]+i \omega[C]+i[D]+[K]
$$

Inserting (16) and (20) into (18), we have

$$
([\Lambda]+[\tilde{\nu}])\{\tilde{Y}\}=\{F\}
$$

leading to the final compact representation of the nonlinear function:

$$
[\tilde{\Lambda}]\{\tilde{Y}\}=\{F\}, \quad \text { where: }[\tilde{\Lambda}]=[\Lambda]+[\tilde{\nu}]
$$

where $[\tilde{\Lambda}]$ is a composite matrix, enclosing linear and nonlinear coefficients and it is formulated for the current state $[\dot{y}, y, \omega]$. It can be considered the system matrix. Obtaining the determinant roots of $[\tilde{\Lambda}]$ yields the frequency-dependent nonlinear natural frequencies and damping ratios. The system response $\{\tilde{Y}\}$ can be obtained solving (27).

The solution of (27) can be used to adjust and/or update the parameters in numerical models obtained through the finite-Element method by comparing numerical and experimental results [12]. Satisfactory results were obtained using the genetic algorithms method [13] to make the approximation of the numeric and experimental values. The GA's parameters used in this application are mutation rate $=0.02$, population size $=50$, and number of generations $=5000$. The objective function is defined by

$$
f=\sum_{i=1}^{n p}\left|P a a_{\mathrm{exp}}-P a a_{\mathrm{FEM}}\right|,
$$

where $P a a_{\exp }$ is the acceleration ratio (acceleration of the reference sensor/acceleration of the accelerometer on the cable); $P a a_{\mathrm{FEM}}$ is the same acceleration ratio estimated using GA; $n p$ is the number of points (normally $n p=47$ ).

\section{Results}

Figure 2 shows the schematic representation of the machine cam used in the experimental testing of the Stockbridge damper with controlled oscillation (displacement). The experimental data are obtained through three accelerometers placed in the half sample. One accelerometer was placed in the center (A2) and another at the end of the messenger wire (A3). A third accelerometer (reference) was placed on the connecting rod (A1).

Figure 3 shows the cam machine with Stockbridge damper coupled and two accelerometers positioned on the weight. The data acquisition system is composed of a signal analyzer (HP 3567) and modal accelerometers (PCB 333AX, sensitivity $\approx 100 \mathrm{mv} / \mathrm{g}$ ). The cam machine with radial flatfaced follower is manually operated.

Five different disk cams with eccentricities of $0.25,0.5$, $0.75,1.25$, and $1.5 \mathrm{~mm}$ were used. The tests were carried out varying the excitation frequency between 5 and $17 \mathrm{~Hz}$ with increments of $0.25 \mathrm{~Hz}$. This lower frequency range was used due to the mechanical limitation of the excitation system. Figures 4 and 5 show the experimental curves of the acceleration ratio obtained for the accelerometers placed in 


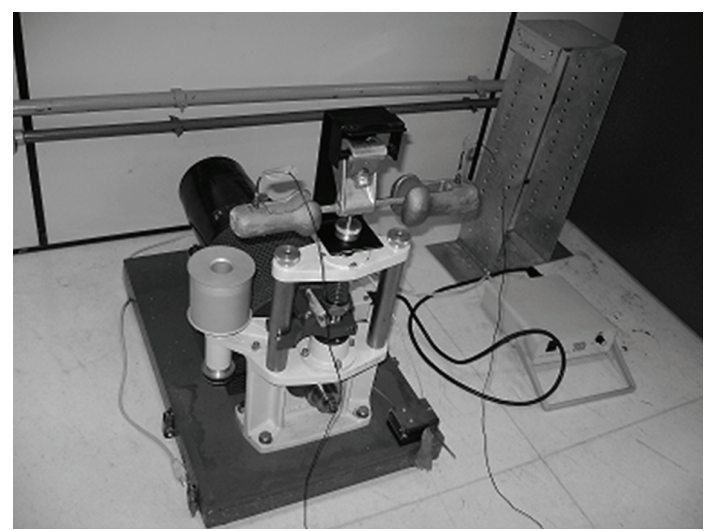

FIgURe 3: Cam machine with Stockbridge damper coupled.

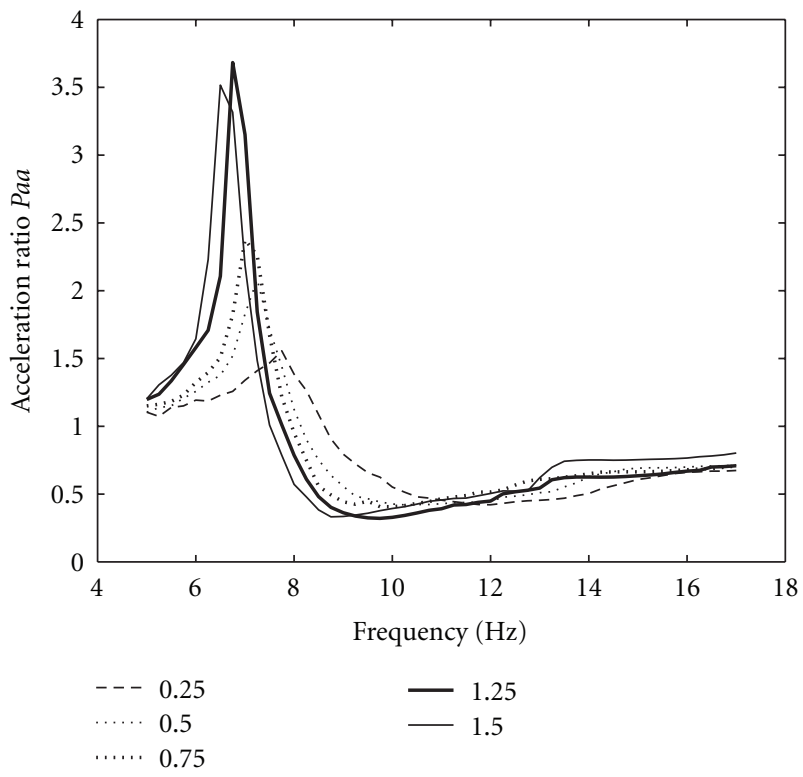

FIgURE 4: Acceleration ratio curves of accelerometer 1.

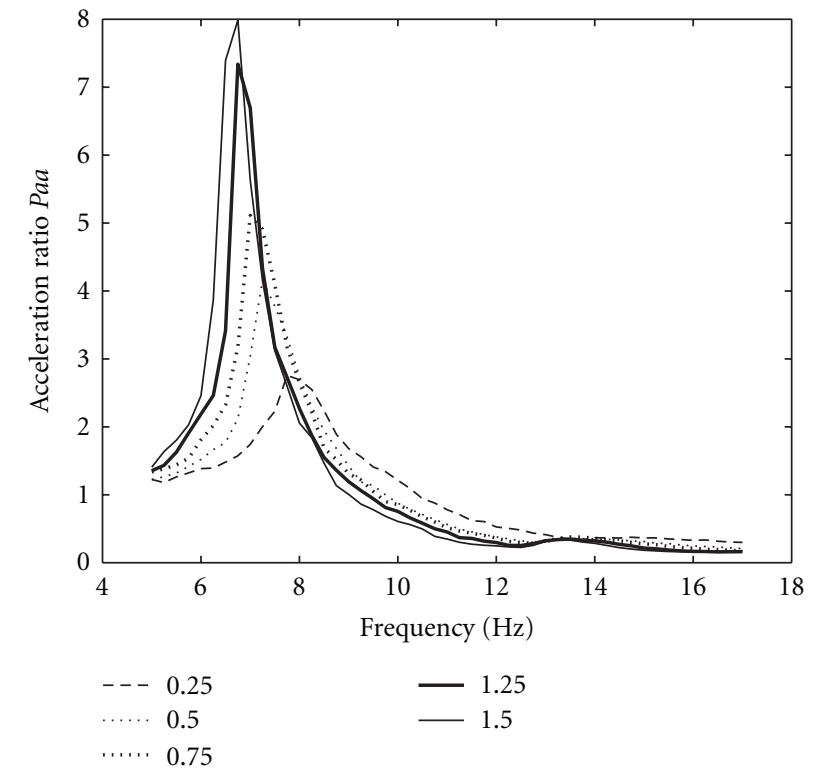

Figure 5: Acceleration ratio curves of accelerometer 2. the center (accelerometer 1) and at the end (accelerometer 2) of the messenger wire. The natural frequency changes with the motion amplitude can be noticed. The frequency variation is inversely proportional to the motion amplitude. Figures 6(a) and 6(b) show the variation of the natural frequency and the damping ratio (adjusted with the linear system) versus the amplitude of the base excitation. It can be noted that parameters, the natural frequency, and the damping ratio vary inversely with the increase of the base motion amplitude. López and Venegas [6] found slightly different results in experimental tests.

The linear and nonlinear parameters used in the numerical models were adjusted considering five different cam profiles. The results are shown in Table 1. The linear parameters adjusted are the Young's modulus $E$ and the loss factor $\eta$ according to (2).

To simulate the hysteretic damping of the system, it was considered $\eta=\eta_{i} / \omega$ (frequency dependent parameter). For the nonlinear system, these two parameters and the other nonlinear parameters of stiffness $\left(\beta_{1}\right.$ and $\left.\beta_{2}\right)$ and damping $\left(\gamma_{1}\right.$ and $\left.\gamma_{2}\right)$, described in (16) and (18) using the procedure described in [8] and only considering the main terms of the nonlinear stiffness and damping matrixes (main diagonal), were adjusted. The error column (Table 1) represents the sum of the absolute errors (difference between the linear and nonlinear FRF) obtained for a limited range of frequencies (approximately $2 \mathrm{~Hz}$ in the resonance region) divided by the number of points.

Figure 7(a) shows the reference base displacement curve obtained in a conventional testing using an electromechanical shaker for excitation. The base displacement variation with the excitation frequency can be noted. The real acceleration measured at Stockbridge damper was considered as input for the mathematical system (Figure 7(b)).

Figures 8(a) and 8(b) show the fitted curves of $E I$ (bending stiffness) and $\eta$ (loss factor) as a function of the base 


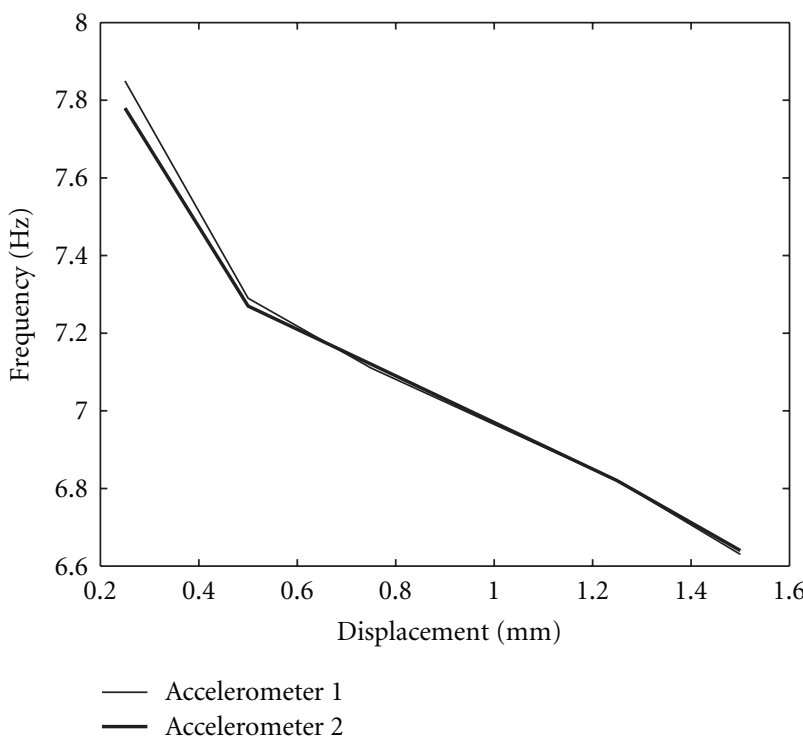

(a)

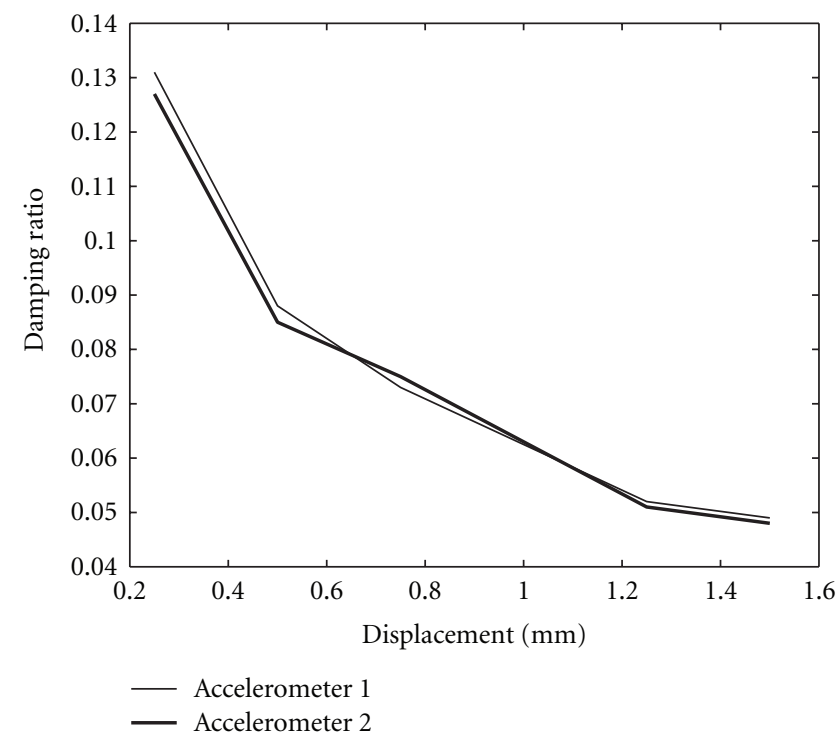

(b)

Figure 6: Curves of natural frequencies and damping ratio versus base displacement.

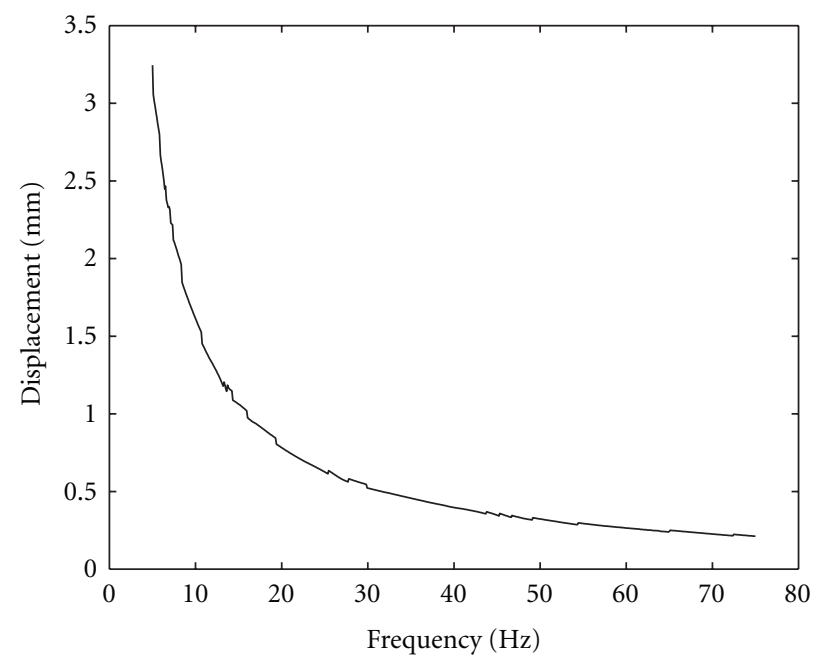

(a)

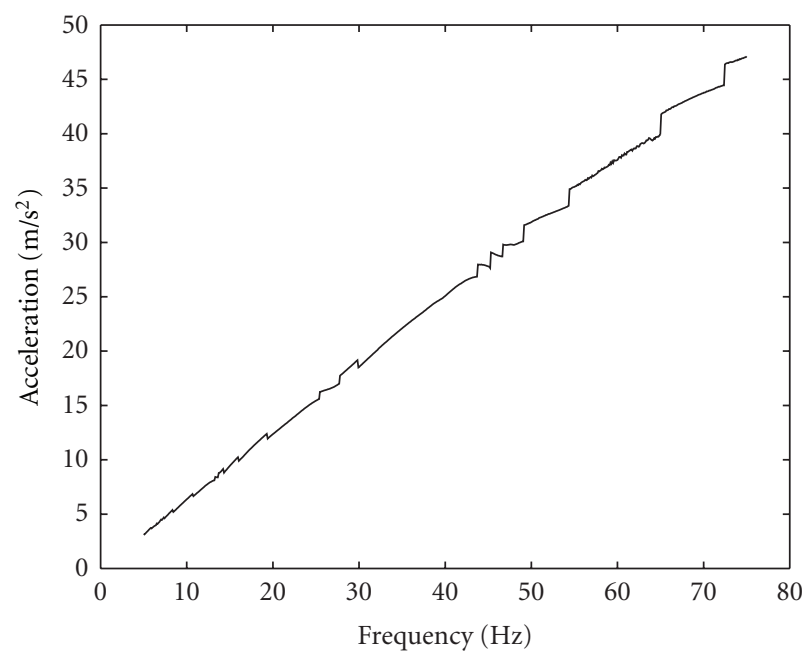

(b)

FIgURE 7: Base displacement and acceleration.

TABLE 1: Parameters adjusted for the linear and nonlinear systems

\begin{tabular}{|c|c|c|c|c|c|c|c|c|}
\hline \multicolumn{2}{|c|}{ Eccentricity (mm) } & \multirow{2}{*}{$\begin{array}{c}\text { Absolute error } \\
0.589\end{array}$} & \multirow{2}{*}{$\frac{E I\left(\mathrm{Nm}^{2}\right)}{1.90}$} & \multirow{2}{*}{$\begin{array}{c}\eta_{i} \\
9.82\end{array}$} & \multirow[t]{2}{*}{$\beta_{1}\left(\mathrm{~N} / \mathrm{m}^{3}\right)$} & \multirow[t]{2}{*}{$\beta_{2}\left(\mathrm{~N} / \mathrm{m}^{3}\right)$} & \multirow[t]{2}{*}{$\gamma_{1}(\mathrm{~N})$} & \multirow[t]{2}{*}{$\gamma_{2}(\mathrm{~N})$} \\
\hline 025 & linear & & & & & & & \\
\hline $0.2 J$ & nonlinear & 0.600 & 1.98 & 11.46 & $-7.9 \mathrm{e} 9$ & -0.0036 & -0.0264 & -0.0502 \\
\hline \multirow{2}{*}{0.5} & linear & 0.454 & 1.74 & 6.21 & & & & \\
\hline & nonlinear & 0.480 & 1.69 & 7.68 & $-9.59 \mathrm{e} 8$ & -0.0308 & -0.0416 & -0.0964 \\
\hline \multirow{2}{*}{0.75} & linear & 0.496 & 1.65 & 4.40 & & & & \\
\hline & nonlinear & 0.464 & 1.67 & 6.57 & $-4.59 \mathrm{e} 8$ & -0.0077 & -0.0445 & -0.0084 \\
\hline \multirow{2}{*}{1.25} & linear & 0.568 & 1.52 & 2.94 & & & & \\
\hline & nonlinear & 0.569 & 1.54 & 3.72 & $-1.67 \mathrm{e} 8$ & -0.0182 & -0.0490 & -0.0537 \\
\hline \multirow{2}{*}{1.5} & linear & 0.828 & 1.43 & 2.56 & & & & \\
\hline & nonlinear & 0.797 & 1.08 & 11.24 & $-5.20 \mathrm{e} 8$ & -0.1601 & -0.0147 & -0.0996 \\
\hline
\end{tabular}




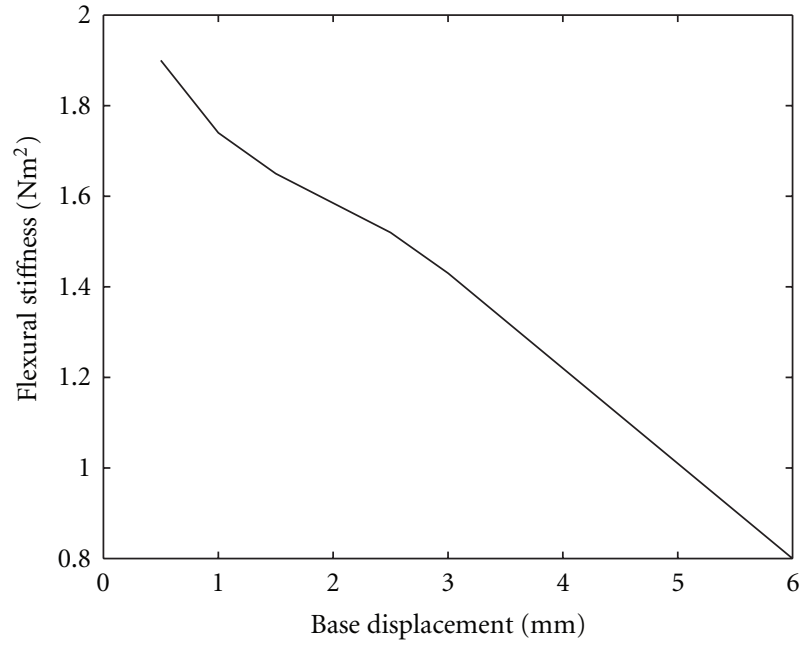

(a)

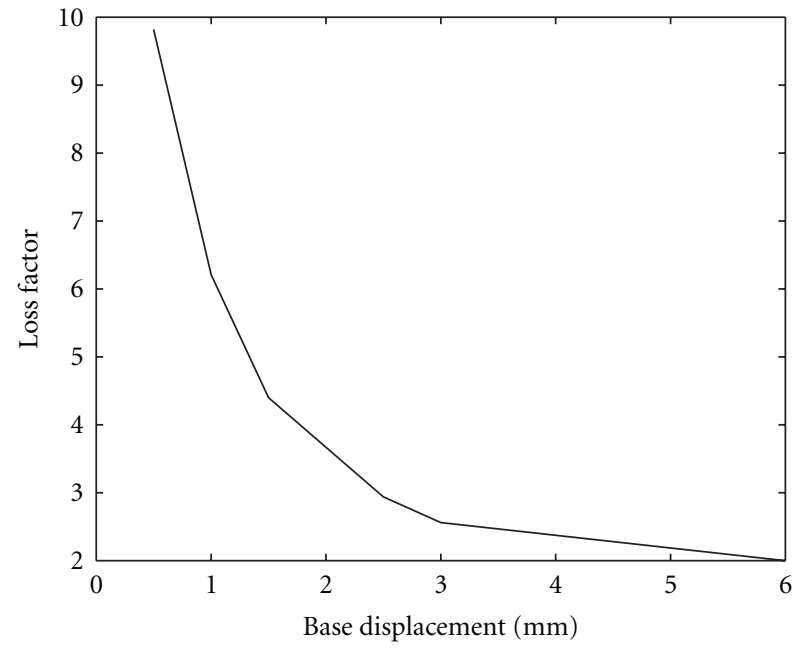

(b)

FIgURE 8: Curve of the flexural stiffness and loss factor versus base displacement.

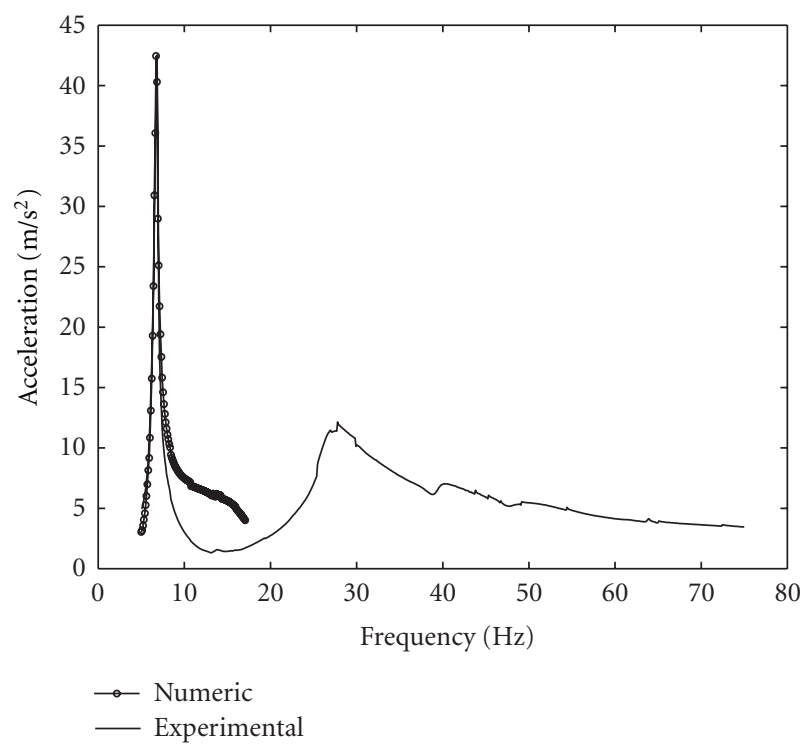

Figure 9: Cable acceleration.

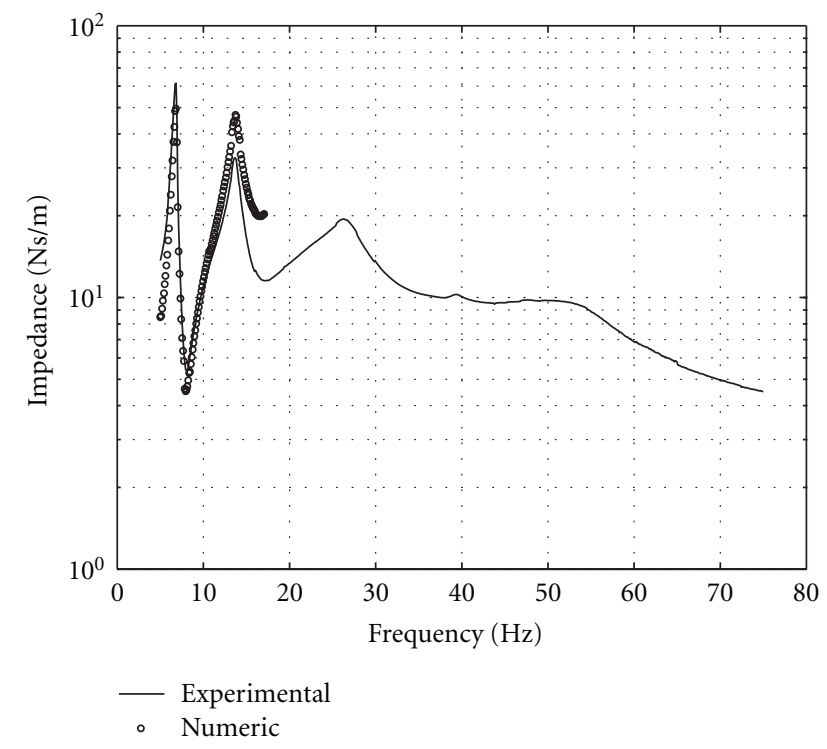

Figure 10: Impedance curves. motion amplitude. These curves are used as a reference for adjusting the Stockbridge damper parameters tested in an electromechanical shaker.

Figure 9 shows the experimental and adjusted cable acceleration curves using a linear numerical model. The curves present good agreement for $f<=10 \mathrm{~Hz}$ (region of the first vibration mode). As the frequency testing is lower than $17 \mathrm{~Hz}$, it is not possible to extrapolate the results for $f>17 \mathrm{~Hz}$.

Figure 10 shows a typical experimentally measured impedance curve and the corresponding numeric curve fitted (linear model) with the parameters shown in Figure 8. It is possible to notice the good agreement between the numeric and experimental curves.

\section{Conclusions}

The results showed that the natural frequency of the system varies with the amplitude of the excitation motion, that is, the higher the motion amplitude is the lower the natural frequency gets. The same behavior was verified for the damping ratio of the system, that is, the higher the motion amplitude is the lower the damping ratio gets.

It was verified that the real and imaginary parts of the complex Young modulus decrease with the motion amplitude increase when the parameters are fitted for a linear system.

For the frequencies range $5 \leq f \leq 17 \mathrm{~Hz}$ and the motion displacement smaller than $3 \mathrm{~mm}$, the system response with 
nonlinear parameters did not show improvements in relation to the linear system response.

As the test was done with a full Stockbridge damper, it is possible that the smallest cable and the smallest mass have influenced the results. The mathematical model was based only on the Stockbridge damper half model containing the largest weight.

When the tests are performed with constant base motion amplitude, it is possible to fit a single value of the parameters according to the motion amplitude.

When the tests are carried out using an electromechanical shaker, the motion amplitude is varied according to the excitation frequency. In this case, the fitted parameters are frequency dependent.

The typical numerical and experimental impedance curves present good agreement.

\section{Acknowledgment}

The authors gratefully acknowledge the support for this work provided by the Brazilian Science Foundation CNPq.

\section{References}

[1] A. Nawrocki and M. Labrosse, "A finite element model for simple straight wire rope strands," Computers and Structures, vol. 77, no. 4, pp. 345-359, 2000.

[2] H. Verma, The Stockbridge damper as a continuous hysteric system in single overhead transmission lines [M.S. thesis], Indian Institute of Technology Bombay, 2002.

[3] Z. H. Zhu and S. A. Meguid, "Elastodynamic analysis of low tension cables using a new curved beam element," International Journal of Solids and Structures, vol. 43, no. 6, pp. 1490-1504, 2006.

[4] Z. H. Zhu and S. A. Meguid, "Modeling and simulation of aerial refueling by finite element method," International Journal of Solids and Structures, vol. 44, no. 24, pp. 8057-8073, 2007.

[5] Z. H. Zhu and S. A. Meguid, "Nonlinear FE-based investigation of flexural damping of slacking wire cables," International Journal of Solids and Structures, vol. 44, no. 16, pp. 5122-5132, 2007.

[6] A. Lara-López and J. C. Venegas, "Endurance of dampers for electric conductors," International Journal of Fatigue, vol. 23, no. 1, pp. 21-28, 2001.

[7] G. Kerschen, K. Worden, A. F. Vakakis, and J. C. Golinval, "Past, present and future of nonlinear system identification in structural dynamics," Mechanical Systems and Signal Processing, vol. 20, no. 3, pp. 505-592, 2006.

[8] H. R. E. Siller, Non-linear modal analysis methods for engineering structures [Ph.D. thesis], Imperial College London/University of London, 2004.

[9] G. Baldo, A. Bonelli, O. Bursi, and S. Erlicher, "The accuracy of the generalized- $\alpha$ method in the time integration of nonlinear single- and two-DOF forced systems," Computational Mechanics, vol. 38, no. 1, pp. 15-31, 2006.

[10] D. Akgün and I. Çankaya, "Frequency response investigations of multi-input multi-output nonlinear systems using automated symbolic harmonic balance method," Nonlinear Dynamics, vol. 61, no. 4, pp. 803-818, 2010.

[11] R. K. Narisetti, M. Ruzzene, and M. J. Leamy, "Study of wave propagation in strongly nonlinear periodic lattices using a harmonic balance approach," Wave Motion, vol. 49, no. 2, pp. 394-210, 2012.

[12] H. Grafe, Model updating of large structural dynamics models using measured response functions [Ph.D. thesis], Imperial College London/ University of London, 1998.

[13] W. D. Chang, "An improved real-coded genetic algorithm for parameters estimation of nonlinear systems," Mechanical Systems and Signal Processing, vol. 20, no. 1, pp. 236-246, 2006. 

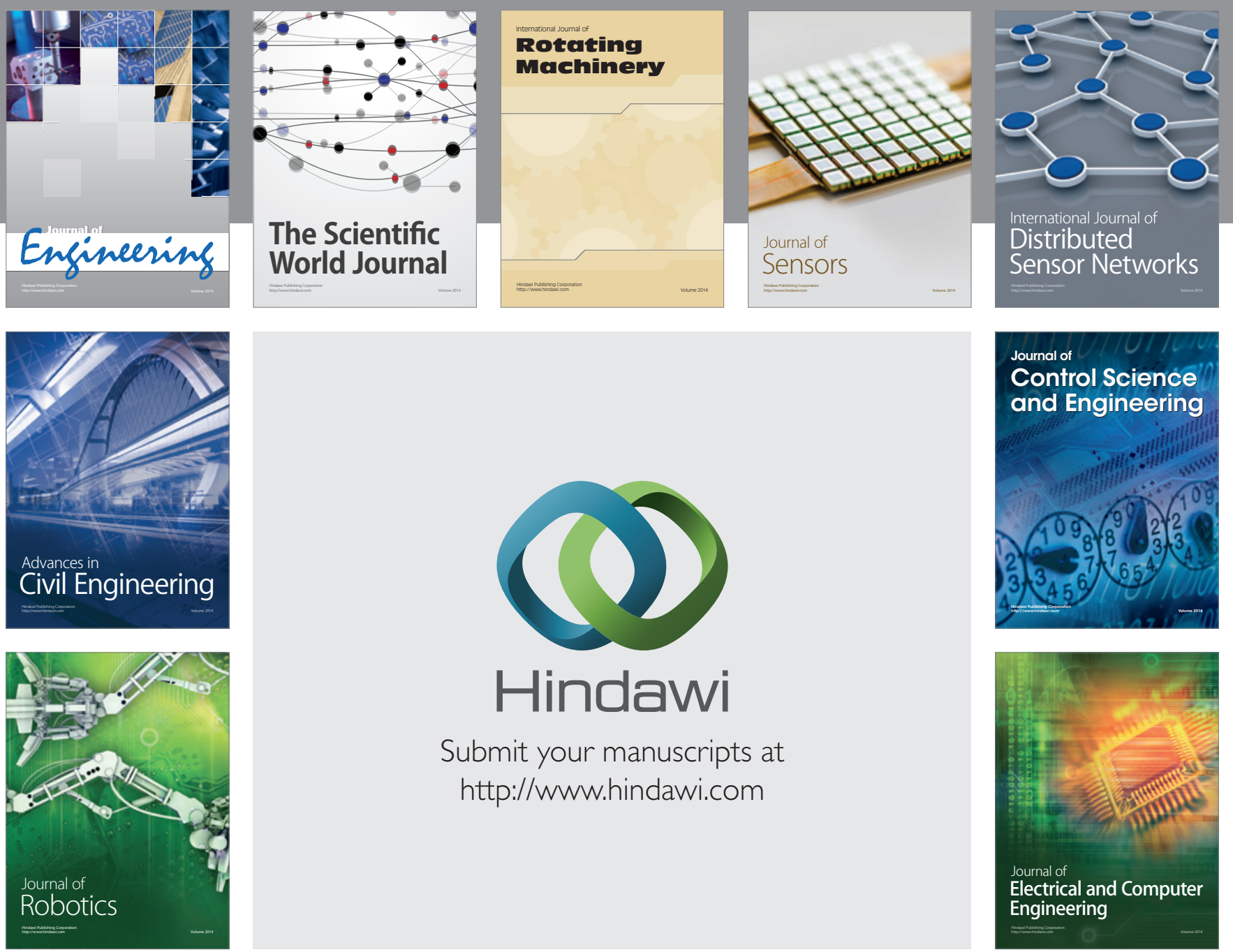

Submit your manuscripts at

http://www.hindawi.com
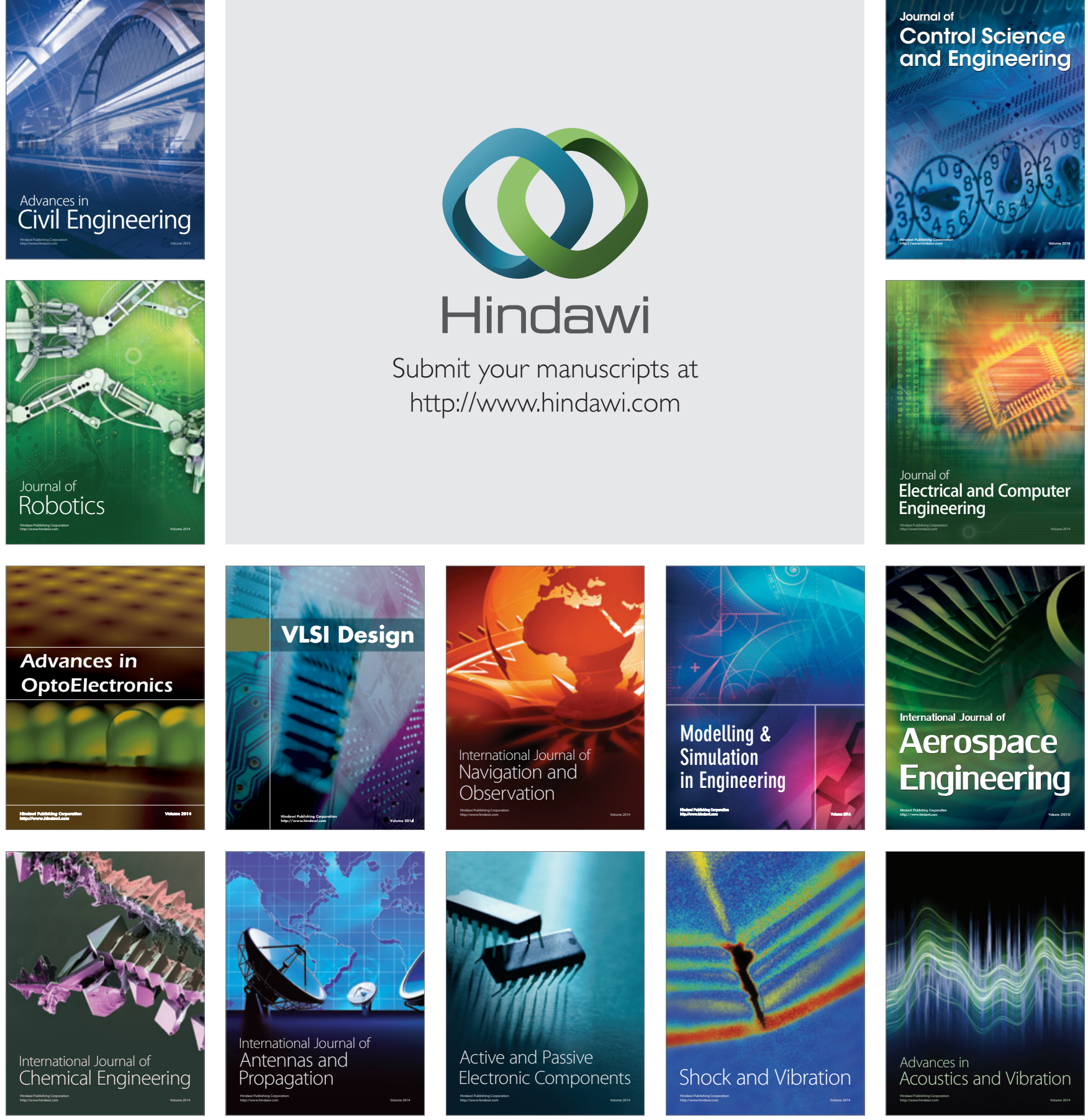